\title{
A 2D Molecular Imprinting Approach to Produce Optical Biosensor Recognition Elements
}

Xiao Li and Scott M. Husson*

Department of Chemical and Biomolecular Engineering, Clemson University, Clemson, SC 29634-0909

AUTHOR EMAIL ADDRESS: shusson@clemson.edu

* To whom correspondence should be addressed. Tel.: +1 (864) 656-4502. Fax: +1 (864) 656-0784. E-mail: shusson@clemson.edu.

\section{Supplemental Information}

Ellipsometry Details. A phase-modulated, spectroscopic ellipsometer (Picometer $^{\mathrm{TM}}$, Beaglehole Instruments) was used that employs a photoelastic birefringence modulator to modulate the light source. Optimized measurement conditions were a wavelength of $632.8 \mathrm{~nm}$ and an incident angle range from $50^{\circ}$ to $80^{\circ}$. Ellipsometric angles $\psi$ and $\Delta$ were measured by changing the angle of incidence and were fitted to a one-layer Cauchy model (Igor Pro. software) to determine the thickness. The refractive index value for monolayers was assumed to be 1.5 [Ulman, 1991]. Three sample spots were taken for each sample in ambient air.

Molecular Structures of Template Molecules. Figure 1S shows the molecular structures for Nع-dansyl-L-lysine (DK) and N,N'-didansyl-L-lysine (DDK). 

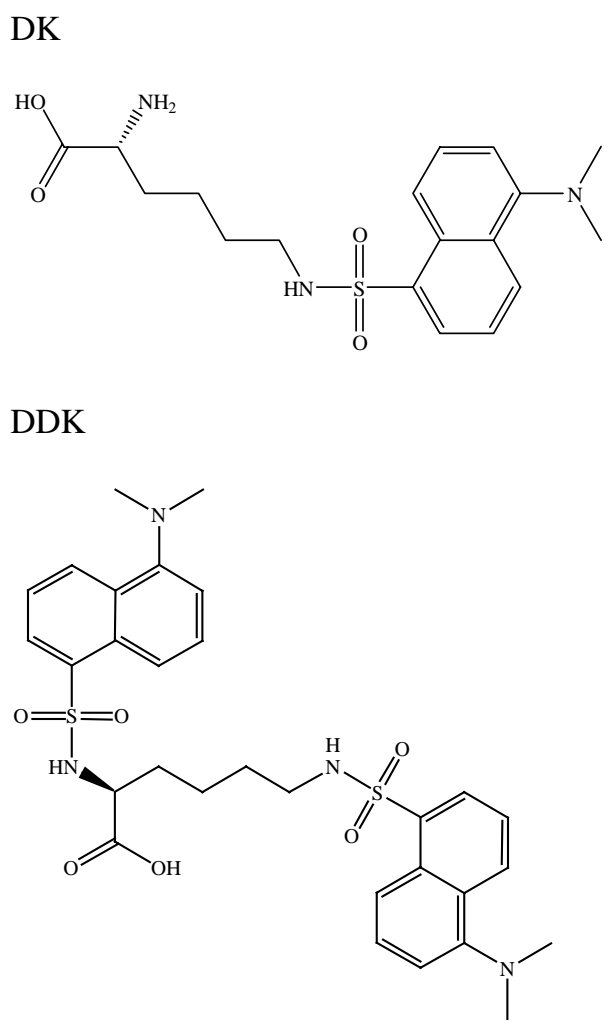

Figure 1S. Chemical structures of DK and DDK.

\section{Reference}

Ulman, A., 1991. Introduction to ultrathin organic films from Langmuir blodgett to self assembly. Academic Press: Boston. 